\title{
Static Nonlinear Aeroelasticity of Flexible Slender Wings in Compressible Flow
}

\author{
Rafael Palacios* and Carlos E.S. Cesnik ${ }^{\dagger}$ \\ The University of Michigan, Ann Arbor, MI, 48109-2140
}

\begin{abstract}
A high-fidelity numerical formulation is presented for the high-speed aeroelastic behavior of slender composite wings. The compressible flow is modeled using the 3-D Euler equations on a deformable mesh, and an asymptotic approximation of the 3-D kinematically-nonlinear equations of elasticity models the anisotropic slender structure. The transfer of the distributed loads and displacements at the fluid-structure interface is based on detailed 3-D representations of the deformed aerodynamic and structural domains. Finally, a time-domain solution is implemented for the closely-coupled fluid-structure interaction problem. This procedure handles the large deflections appearing in very slender wings under aerodynamic loads using a description of the deformation that includes all geometrically-nonlinear effects in the aeroelastic analysis. Using this approach, the static nonlinear aeroelastic response of a 16:1 half-aspect ratio wing is investigated for steady flight conditions. The impact of the detailed 3-D representation of the fluid-structure interface on the aeroelastic response is investigated. For that purpose, numerical results are compared to the representation of the structure using a geometricallynonlinear 1-D beam model.
\end{abstract}

\section{Introduction}

$\mathrm{D}$ ESIGNING high-altitude long-endurance unmanned aerial vehicles (HALE UAVs) demands high levels of aerodynamic and structural efficiency. Among current research efforts in this topic, the SensorCraft program $^{1}$ is exploring different mechanisms to improve HALE aircraft performance, such as adaptive structures and flow control techniques. All proposed SensorCraft platforms are based on very high aspect-ratio wings to achieve the desired high lift-to-drag ratio with low maneuverability requirements. From a structural viewpoint this leads to very flexible constructions that 1) will be subject to large deflections under flight loads and 2) will present important rigid-elastic coupling effects. This paper takes on the first point: the development of refined numerical methodology for high-fidelity aeroelastic analysis of very flexible wings. This is accomplished here by coupling a CFD solution on a moving grid and a kinematically-nonlinear structural one. The nonlinear framework is solved by time-marching the equations in the time domain, although for the present static aeroelastic studies, only the steady-state converged solution is of importance.

The structural formulation takes advantage of the wing slenderness to simplify the modeling and solution processes. Slender structures have a dominant spatial direction, and they can be effectively modeled using a dimensional reduction of the problem to a reference line along that direction. The usual approach stems from Cosserat's beam model ${ }^{2}$ : the structure is represented by a deformable curve in space with cross sections that move rigidly with it. Elastic properties are then estimated and the deformation of the curve is analyzed using the tools of differential geometry, including a kinematical description through a set of intrinsic quantities (strain measures). Successive works (Kirchhoff ${ }^{3}$, Reissner ${ }^{4}$, $\mathrm{Simo}^{5}$, Hodges $^{6}$ ) have developed the geometrically-exact intrinsic equations for the dynamics of beams. Garcia et al. ${ }^{7}$ and Smith et al. ${ }^{8}$ have coupled then with Euler/Navier Stokes solutions of the flowfield for fully nonlinear aeroelastic

\footnotetext{
* Graduate Student (palacios@fulbrightweb.org), Department of Aerospace Engineering, F.-X. Bagnaud Fellow

† Associate Professor (cesnik@umich.edu), Department of Aerospace Engineering, Associate Fellow, AIAA.

Copyright $₫ 2005$ by Rafael Palacios and Carlos E. S. Cesnik. Published by the American Institute of Aeronautics and Astronautics, Inc., with permission.
} 
analysis of slender wings. Beam models result in 1-D idealizations of the structure that capture the average deformations of the 3-D solid. However, they do not provide much information of the actual elastic solution in the 3-D domain, which is needed in high-fidelity fluid-structure interaction problems.

To overcome this, the authors ${ }^{9-11}$ have developed a solution method of the general problem of electro-thermoelasticity in slender 3-D domains that provides detail information about the 3-D solution in a recovery process after the 1-D problem. This was achieved by the identification of two different spatial scales in the solution: a long-scale contribution associated to the variations along the dominant dimension in the structure, and a short-scale contribution after the local equilibrium at the cross section. It was shown using an asymptotic solution procedure ${ }^{11}$ that both problems can be recursively solved to provide a full description of the 3-D elastic structure. This paper presents the application of the numerical methodology of Ref. 11 to define the solid side of a fluid-structure interaction problem. Although the 3-D structural analysis was originally developed to also account for distributed anisotropic piezocomposites actuators and sensors, this feature is not used in this paper. The fluid is modeled by the Euler equations in the ENS3DAE solver ${ }^{12}$, and the interfacing and interpolation between both modules is managed by MDICE ${ }^{13}$. Results are presented on a straight nontapered wing of aspect ratio 16 and with a NACA0012 airfoil.

\section{Methodology}

The fluid-structure interaction procedure uses a closely-coupled methodology, according to the definition of Smith et $a l .{ }^{14}$. There is a transfer of information between both modules at each time step (or iteration): surface loads from the aerodynamics to the structure, and surface deformation from the structure to the aerodynamics. Figure 1 shows a typical closely coupling procedure between Computational Fluid Dynamics (CFD) and Computational Structural Mechanics (CSM) modules in a steady state analysis with large displacements. Internal sub-iterations are usually necessary in both the CFD and the CSM solutions to achieve convergence of their nonlinear equations. A relaxation parameter is also needed to facilitate converge under large structural deflections.

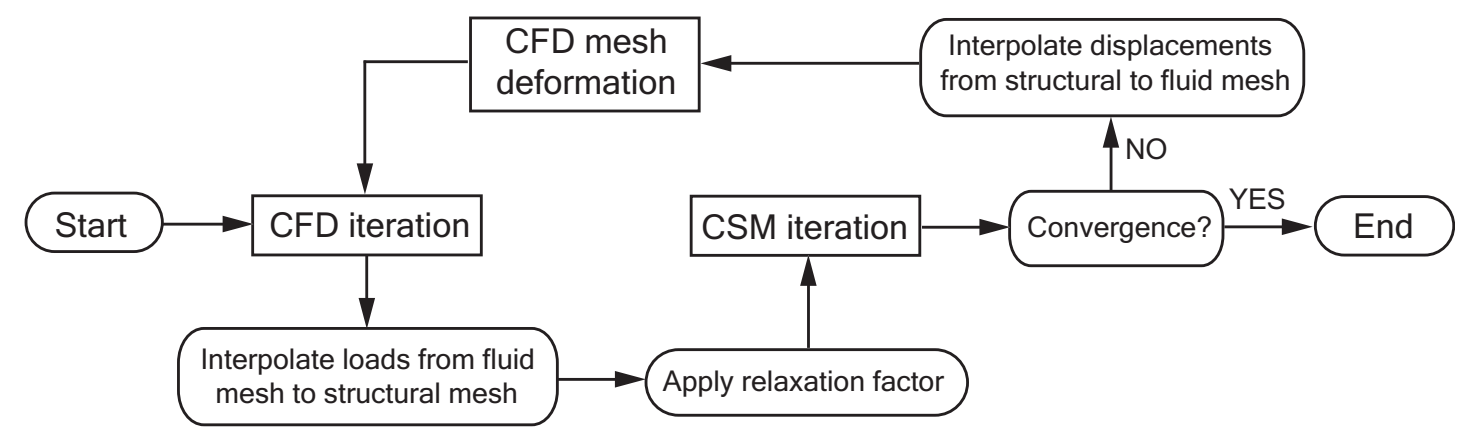

Figure 1. Static aeroelastic coupling procedure with large deflections

\section{A. Structural Module}

A high-fidelity reduced model of the structure is obtained by a mathematically-rigorous process of asymptotic reduction of the general electrothermoelastic anisotropic slender solid ${ }^{9-11}$. The asymptotic solution method splits the problem into i) a linear analysis over the cross section (UM/VABS, Variational-Asymptotic Beam Section analysis ${ }^{9,15}$ ), in which a finite-element procedure solves the cross-sectional displacement and electric field to minimize the local energy state; ii) a long-scale problem with a kinematically-exact one-dimensional formulation of the dynamics of the reduced structure (NLABS, Nonlinear Active Beam Solver ${ }^{10}$ ). The solution process is schematically represented in Figure 2. For this 1-D problem, a first-order mixed formulation is defined from the intrinsic equations of motion, which is solved using a finite-element discretization. In general, the dynamics at the reference line can be expressed in symbolic form as 


$$
\mathrm{A}(\mathrm{X}) \cdot \dot{\mathrm{X}}+\mathrm{S}\left(\mathrm{X}, \hat{\mathrm{X}}_{x=0}, \hat{\mathrm{X}}_{x=l}\right)=\mathrm{L}(\mathrm{X})
$$

where $\mathrm{A}$ is the inertia matrix operator, $\mathrm{S}$ is the structural operator, and $\mathrm{L}$ is a force operator that includes the aerodynamic loads. $\hat{\mathrm{X}}$ are the boundary values of the 1-D state vector $\mathrm{X}$ at the ends of the 1 -D domain ( $x=0$ and $x=l$ ). The definition of the problem is completed with the appropriate spatial boundary and initial conditions at $t=t_{o}$, which are also obtained from a variational setup

$$
B C\left(\hat{\mathrm{X}}_{x=0}(t)\right)=0, B C\left(\hat{\mathrm{X}}_{x=l}(t)\right)=0 \text {, and } \mathrm{X}\left(t_{0}\right)=\mathrm{X}_{0}
$$

An implicit time-domain integration algorithm is finally used on this system of differential-algebraic equations in X, $\hat{\mathrm{X}}_{x=0}$, and $\hat{\mathrm{X}}_{x=l}$. Although independently analyzed, the cross-sectional and longitudinal problems yield together a full description of the solution in the original 3-D domain ${ }^{11}$. This is particularly important in the generation of the solid side of an aeroelastic model: the interface of the structural model consists of the actual wetted surfaces of the vehicle, without extrapolations from the motion of the reference line, such as those described by Brown ${ }^{16}$. Moreover, the present formulation can also capture local effects in the deformation field, such as camber bending, using a Ritz-like approximation of the local warping displacement field (finite-section deformation modes ${ }^{10,11}$ ).

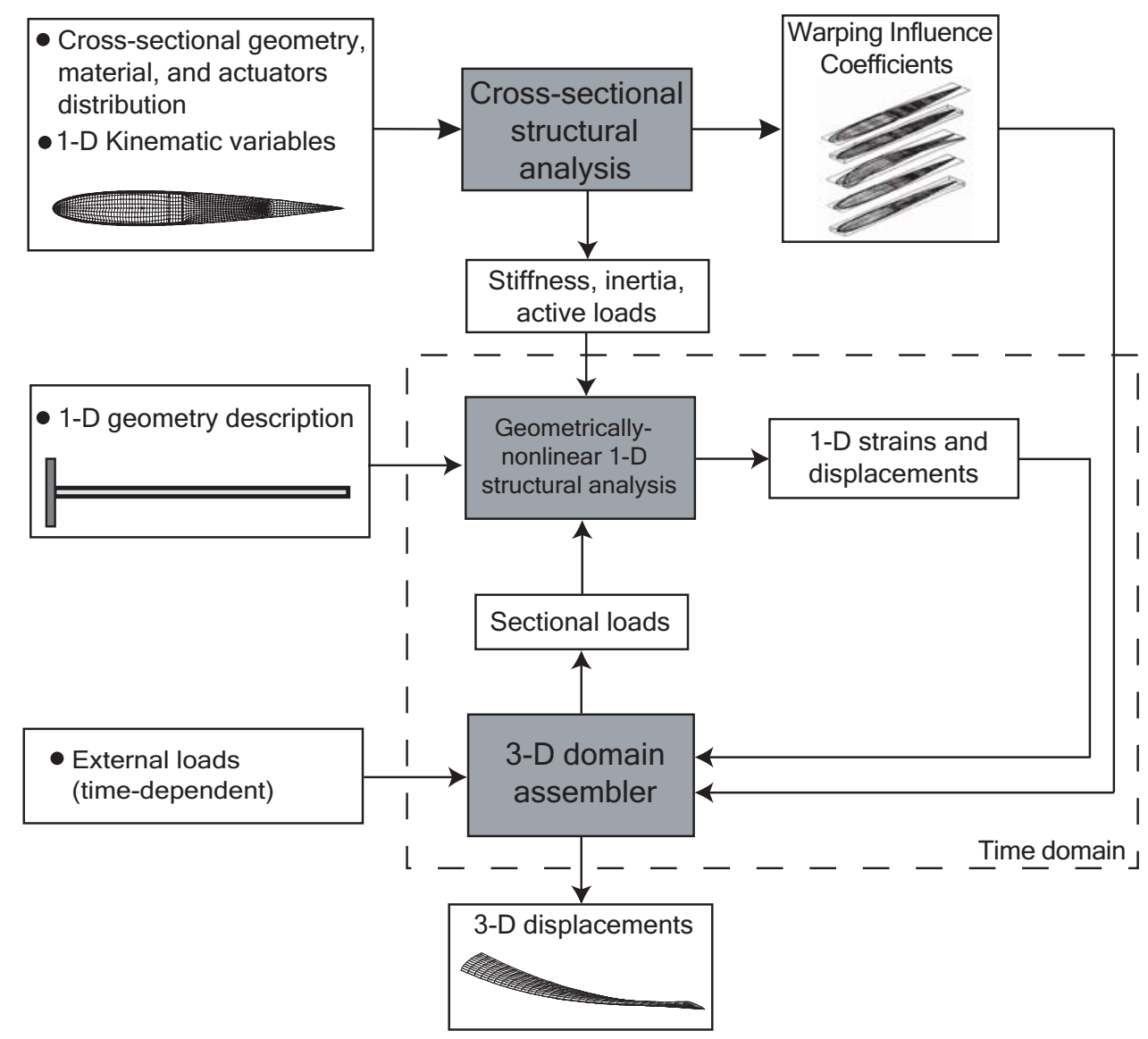

Figure 2. Three-step solution to the nonlinear dynamics of 3-D slender structures 


\section{B. Aerodynamic Model}

In this work, Euler equations are used to model the aerodynamic loads. This selection is made as a compromise between 1) the desired high-fidelity characteristics for the aerodynamic module, and 2) the necessary flexibility for the development of a proof-of-concept application. Therefore, it is assumed that the flowfield is inviscid and non-heatconducting. For attached flows around bodies, these equations provide a good approximation of the pressure field and, therefore, of the lift characteristics, but only induced drag is predicted. The implementation in the ENS3DAE (Euler/Navier-Stokes 3-Dimensional Aeroelastic method ${ }^{12}$ ) solver has been chosen for the analysis. This module solves the full Reynolds-Averaged Navier-Stokes equations in time domain. It also solves simplified versions, such as the inviscid equations (Euler) or the thin shear layer approximation. Without loss of generality, the analyses in this work are based on the compressible Euler solution of the code. A computational mesh is generated using a multiblock discretization of the fluid domain. A finite-difference spatial discretization of the equations is then introduced on this computational mesh, and an implicit time-marching algorithm (unconditionally stable) completes the numerical solution scheme. For computational efficiency, the equations are independently solved at each block, with an iterative procedure until values at the interfaces match. The numerical results in the present analyses are obtained with a Gauss-Seidel algorithm with central differencing and explicit dissipation. The steady-state analyses use a spatially-varying time step for improved convergence.

ENS3DAE also provides a grid update algorithm for use in aeroelastic simulations. For robustness, the method is restricted to block H-grids, and it has been tailored to preserve the quality of the initial grid in the deformation of complex configurations.

\section{Fluid-Structure Interface Module}

The integration of the different modules has been done using MDICE (Multi-Disciplinary Computing Environment ${ }^{13}$ ). MDICE is an object-oriented simulation manager developed by CFDRC, in which each module independently communicates with a central controller. As a result, it provides a common interface for different aerodynamic and structural solvers, which can be therefore easily exchanged. In order to generate MDICE-compliant software, the original source codes need to be modified and attached to a MDICE library of routines. The interface between ENS3DAE and MDICE was written at the Air Force Research Laboratories (AFRL), while the interface between UM/NLABS and MDICE has been written for this work. Python-based script syntax provides the necessary flexibility for the control of the simulations. The fluid-structure interface module in MDICE performs an automatic grid alignment between the solid and fluid interface surfaces. It then interpolates forces and displacements using conservation of total force and virtual work, and the projection of nodes from one interface to the other. The algorithms are based on the work of Refs. 14 and 16.

\section{Numerical Results}

To exemplify the proposed methodology and study the effect of higher-order deformations on the aeroelastic response of slender wings, numerical simulations are carried out on a straight wing with a NACA0012 airfoil. It has constant chord $\mathrm{c}=1 \mathrm{~m}$, half-aspect ratio of 16 (semi-span $\mathrm{L}=16 \mathrm{~m}$ ), and one spar at $0.35 \mathrm{c}$. Two structural models are considered: 1) an isotropic model, with material distribution defined such as the elastic axis is at $0.25 \mathrm{c}$, and bending and torsion stiffness of 800 and $1000 \mathrm{kN} \cdot \mathrm{m}^{2}$, respectively; 2) a composite model with individual plies of (S-Glass) material constants $\mathrm{E}_{\mathrm{T}}=43.4 \mathrm{GPa}, \mathrm{E}_{\mathrm{L}}=12.0, \mathrm{G}_{\mathrm{LT}} 3.6 \mathrm{GPa}$, and $v_{\mathrm{LT}}=0.28$ ("L" and "T" are the longitudinal and transversal directions, respectively), and different lay-ups. Skin lay-ups are given in Table 1 inside out, with nominal ply thickness of $0.457 \mathrm{~mm}$. The spar has thickness $1.71 \mathrm{~mm}$ at $0^{\circ}$.

Table 1. Composite wing lay-ups

\begin{tabular}{lcc}
\hline \hline Case & $\begin{array}{c}\text { Lay-up [Upper Skin]-[Lower } \\
\text { Skin] }\end{array}$ & $\begin{array}{c}\text { Elastic } \\
\text { Coupling }\end{array}$ \\
\hline $\mathbf{1}$ & {$[0 / 45 / 0 /-45 / 0]_{\mathrm{S}}[0 / 45 / 0 /-45 / 0]_{S}$} & none \\
$\mathbf{2}$ & {$[0 / 45 / 0 / 45 / 0]_{S}[0 / 45 / 0 / 45 / 0]_{S}$} & extension-twist \\
$\mathbf{3}$ & {$[0 / 45 / 0 / 45 / 0]_{\mathrm{S}}[0 /-45 / 0 /-45 / 0]_{\mathrm{S}}$} & bending-twist \\
\hline \hline
\end{tabular}


Layup 1 is a quasi-isotropic layup, layup 2 presents extension-twist coupling, and layup 3 shows bending-twist coupling. The aerodynamic mesh of Smith et al. ${ }^{8}$ is used for the analyses, which is defined at 20,000-m altitude and for variable angle of attack and airspeed. Figure 3 shows the undeformed aerodynamic mesh, and an example of deformed mesh under large deflections, which corresponds to the isotropic wing at angle of attack $\alpha=2^{\circ}$ and Mach number $\mathbf{M}_{\infty}=0.5$. For this case, tip deflection is $16 \%$ of the wing semi-span.
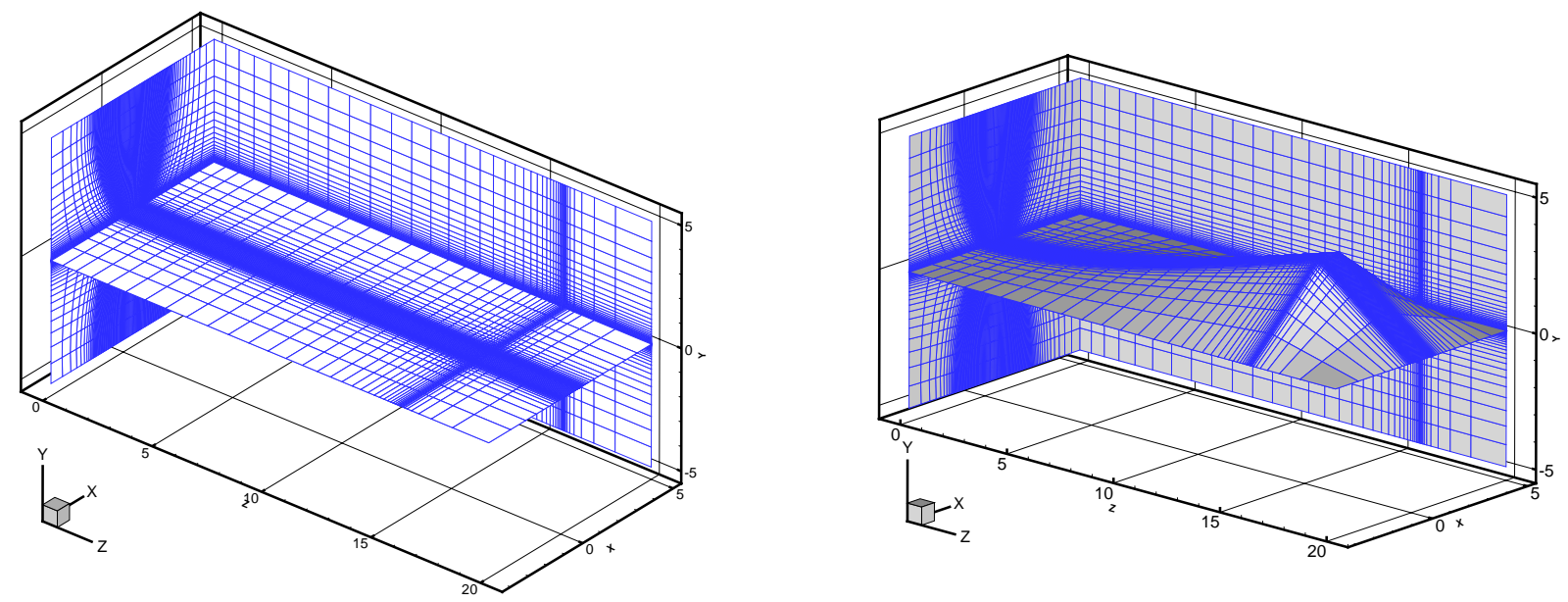

Figure 3. Undeformed and deformed aerodynamic mesh (isotropic wing, $\alpha=2^{0}, M_{\infty}=0.5$ )

\section{A. Geometrically-Nonlinear Wing Deformation}

Geometrically-nonlinear effects in slender wing are noticeable in the loss of total lift due to the spanwise curvature. The wing bends as the velocity increases and the distributed lift behaves as a following force, as shown in Figure 4 for flight at Mach 0.5 .

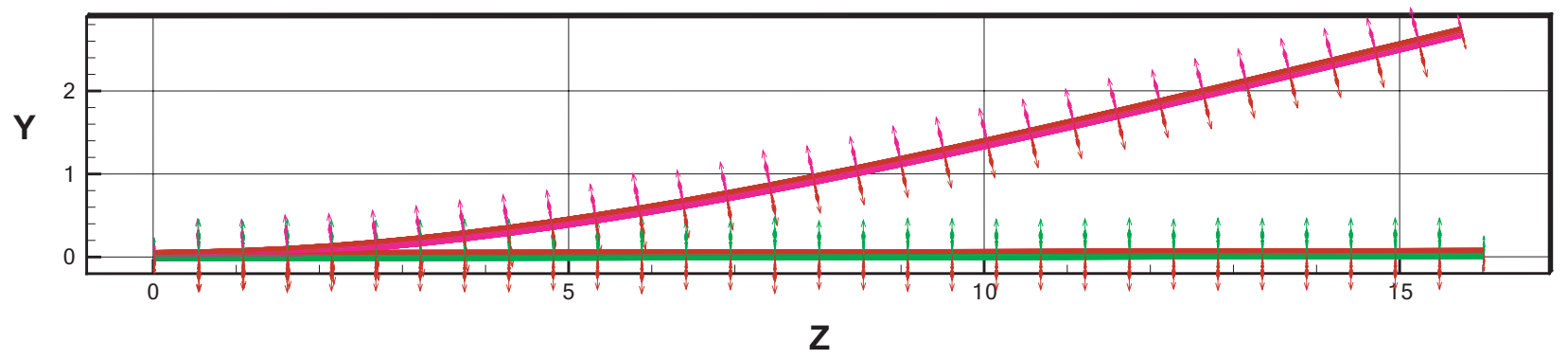

Figure 4. Force distribution in the deformed and undeformed wings (isotropic wing, $\alpha=2^{0}, M_{\infty}=0.5$ )

Figure 5 shows the tip deflection and normalized lift for varying airspeed on the isotropic wing at $\alpha=2^{0}$. Two solutions are shown: linear and nonlinear. The "linear structure" solution contains only linear stiffness effects and the direction of the lift loads is kept constant with respect to the undeformed configuration. The "nonlinear structure" contains the fully nonlinear stiffness, including the change in the loads direction with deformation. In Figure 5, the lift coefficient is normalized with respect to the "linear" case for different Mach numbers.

As expected, the normalized total lift for the nonlinear representation reduces with increase wing deflection due to the bending of the wing (follower force case-see top plot in Figure 5). The corresponding normalized total lift for the "linear structure" does not change. When looking at the two predicted tip equilibrium positions, as shown in Figure 5, there is actually very little difference between the two approaches. This is an interesting coincidence where one error compensates for the other in the linear solution: the geometric stiffening is not present and the load direction is not updated, but the wing bending displacement is very close to the correct one. If the load direction were to be updated, the 
lift would be approximated better but the tip displacement would be off. Although this occurs for this particular structural configuration, it would be interesting to see how this varies with varying dimensions and material properties.
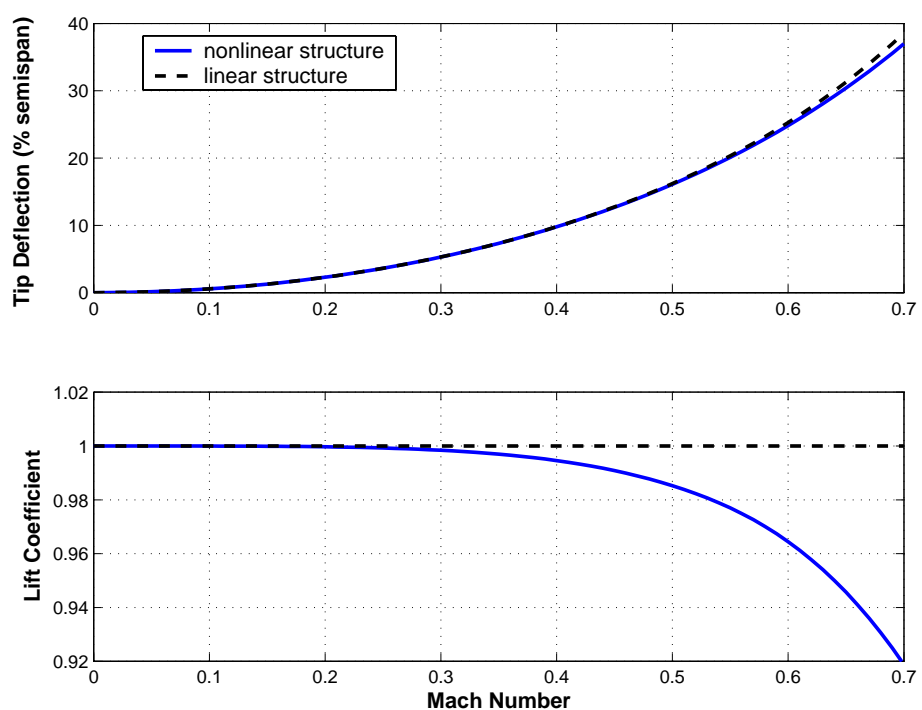

Figure 5. Tip deflection and normalized lift coefficient for increasing speed of the isotropic wing

Considering now the composite wings, different steady state solutions at Mach 0.2, $\alpha=2^{0}$ and Mach $0.7, \alpha=4^{0}$ were computed for the three lay-ups of defined in Table 1 . At $\mathrm{M}_{\infty}=0.7$, the Young's and Shear moduli were multiplied by a factor of 20 with respect to the given nominal material properties so to stiffen the original wing (and keep tip deflections less around $15 \%$ of wing semi-span — see Figure 5). Results at a reference line located at the mid-chord of the wing are shown in Figure 6. In all cases, the plots compare the effect of including the cross-sectional warping deformation in the definition of the wetted surface versus simply assuming rigid cross sections. The correction due to the warping effects is very small in these cases due to relatively stiff design for the given dynamic pressure. Note also that the responses of layups 1 and 2 are expected to be very similar to each other since they only differ due to the presence of extension/twist elastic coupling. But since there are only small axial-wise loading due to the spanwise component of the lift during large wing bending deformations, this term has a small impact in the final equilibrium position. This is only slightly noticeable from the plots, where the two curves are very close to each other.
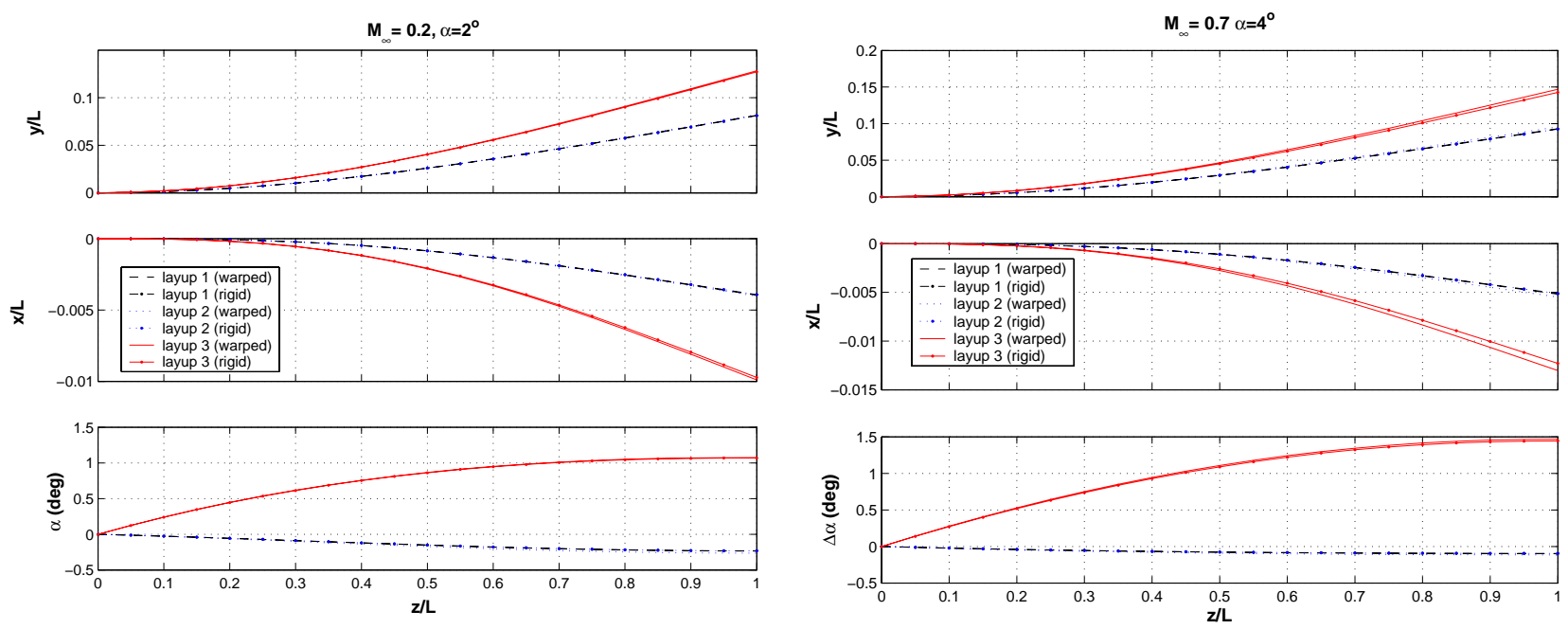

Figure 6. Wing displacements and local increase of angle of attack at the reference line 


\section{B. Compressibility Effects}

At $\mathrm{M}_{\infty}=0.7, \alpha=4^{0}$, transonic effects on the airfoil become important and they have been estimated here by the Euler equations. The local Mach number at the deformed section at $90 \%$ of the semi-span is shown in Figure 7 for two different layups. Due to the relatively thick airfoil (typical of HALE wings), transonic flow is present on the top surface of the wing. Since layup 1 and 3 have different elastic properties, the local elastic twist at $90 \%$ semi-span is different for the two different models. This positions the shock at different chordwise location as seen in Figure 7.

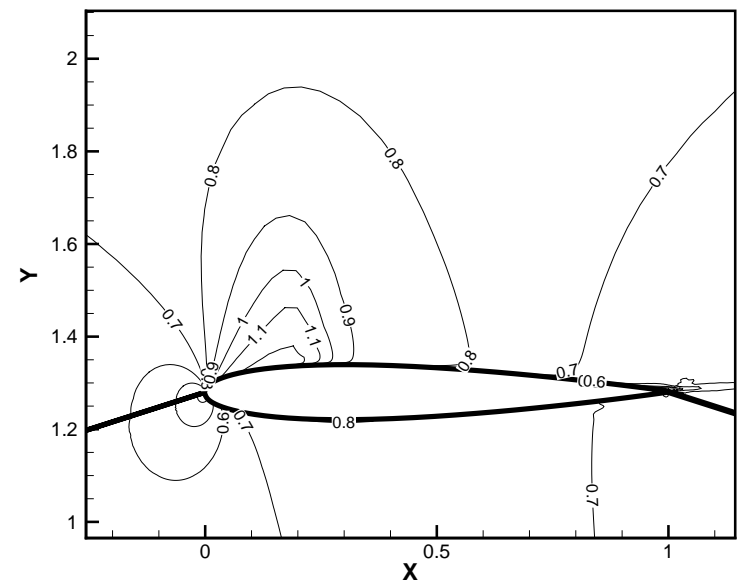

Layup 1

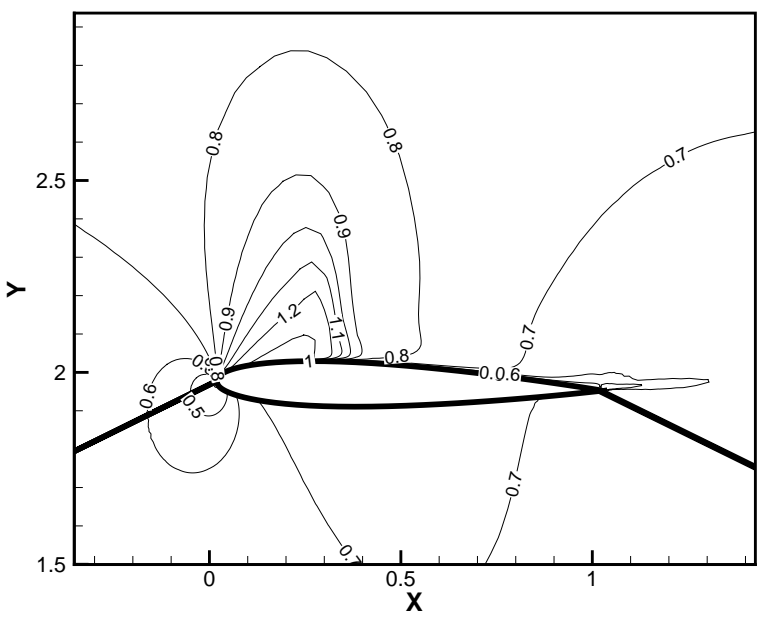

Layup 3

Figure 7. Mach contours at $0.9 \mathrm{~L}$ section $\left(\mathrm{M}_{\infty}=0.7, \alpha=4^{0}\right)$

Consider the same problem as above, that is, the composite wing with layups 1 and 3 at $M_{\infty}=0.7, \alpha=4^{\circ}$, but now with an extra degree of freedom: camber-bending deformation of the cross section (see Figure 8). This results in structural deformations usually only captured by built-up plate-like finite element modeling. In the proposed structural representation, these effects are correctly accounted for as part of the geometrically nonlinear structural formulation (although the cross section deformation is treated in a linear fashion since the local deformations are relatively small). The additional cross-sectional deformation due to the added flexibility, while small compared to the overall wing deformation, can significantly affect the local flow field. This is particular important in this case where transonic effects are presented. Figure 9 shows the local Mach lines for the layups 1 and 3 composite wing when modeled with the additional finite-section mode. For layup 3, when compared with the equivalent plot in Figure 7, one may notice significant shift of the shock to an aft position on the upper side of the airfoil. Similarly, the flow slows down in the lower side of the airfoil when compared to a more rigid model. Although different structural concepts will have different local flexibility, what this examples shows is that even for slender wings the local deformation can make a significant impact on the wing aeroelastic behavior. In this particular case, the lack of chordwise reinforcement and the strong wing bending/camber bending elastic coupling increase the local deformations seem in the plot.

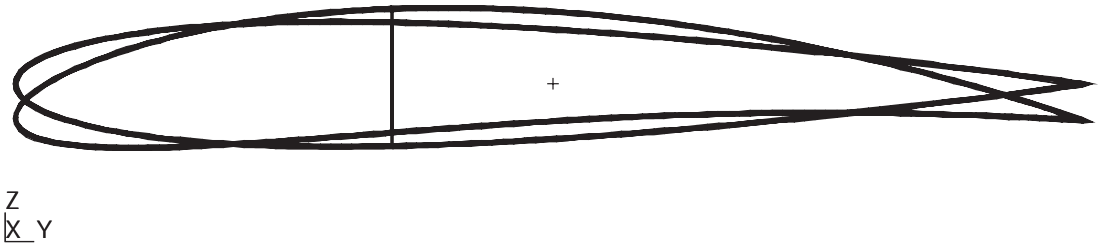

Figure 8. Finite-section mode associated with the camber-bending deformation of the wing 


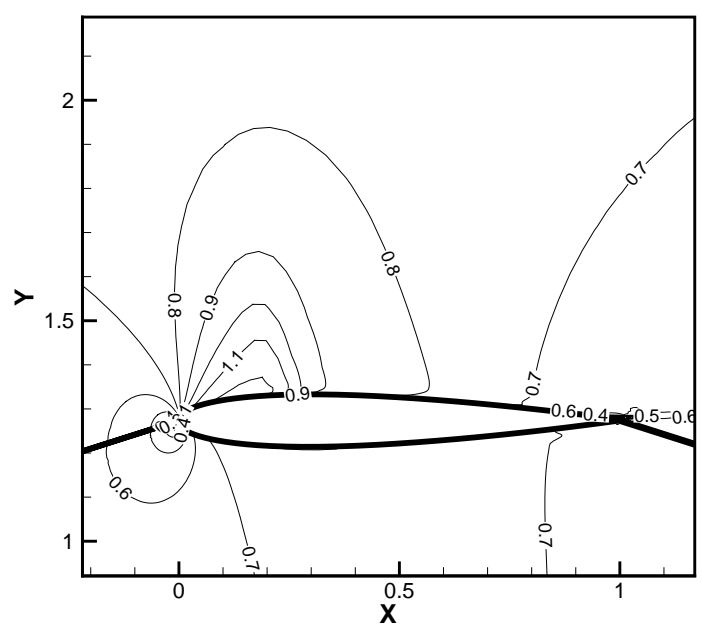

Layup 1

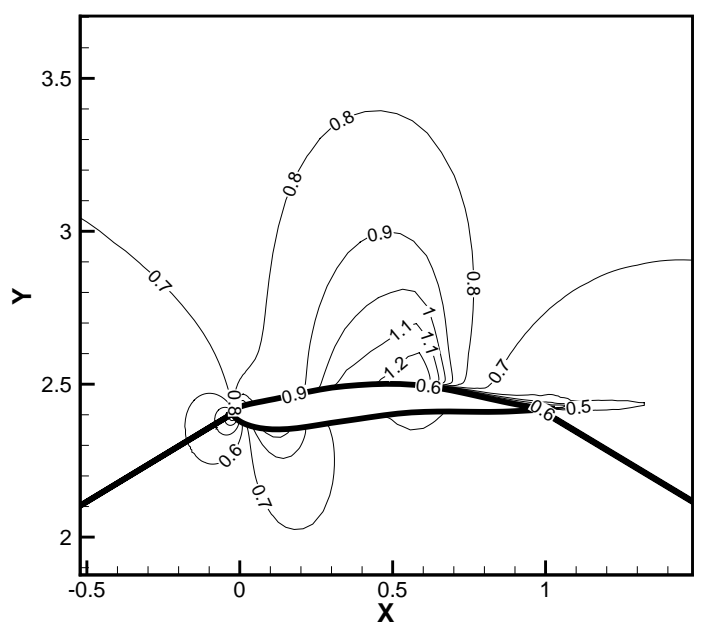

Layup 3

Figure 9. Mach contours with a finite-section mode (camber bending) at $0.9 \mathrm{~L} \operatorname{section}\left(\mathrm{M}_{\infty}=0.7, \alpha=4^{0}\right)$

\section{Relaxation Parameter for Improving Convergence}

In fluid-structure interaction problems involving large deflections, the adequate selection of the relaxation parameter, $\rho$, (see Figure 1) becomes a critical factor to obtain a converged steady-state solution. Large variations in either the wing displacements or the aerodynamic loads within a single iteration may induce numerical instabilities in the coupling procedure. Here the relaxation parameter is applied on the aerodynamic forces before exporting them to the structural surfaces. Figure 10 shows the evolution of the solution for a small relaxation parameter $(\rho=0.02)$, a moderate relaxation parameter $(\rho=0.5)$, and an unrelaxed solution. A small value of the relaxation factor builds up the solution through small increments in load, which was observed to provide a slower but more robust convergence to the steady-state solution. All results in this work were obtained with small values of $\rho$.
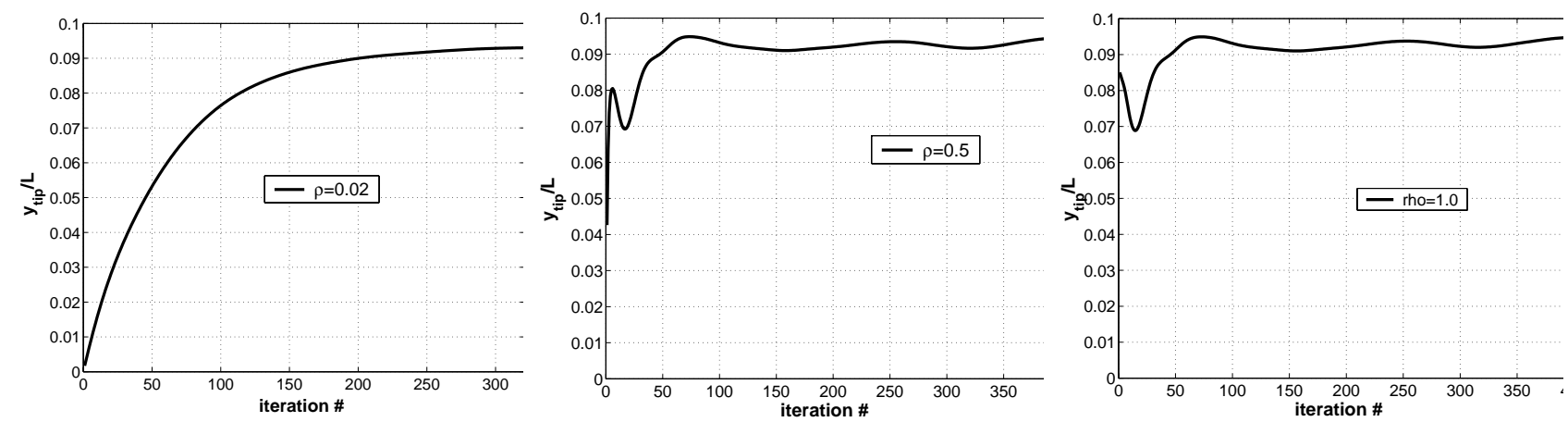

Figure 10. Iterations to convergence for different relaxation parameters $\rho\left(M_{\infty}=0.7, \alpha=4^{0}\right)$ 


\section{Concluding Remarks}

An aeroelastic framework based on nonlinear structural dynamics and compressible unsteady aerodynamics for highfidelity analysis of HALE aircraft was presented. A three-step modeling scheme for the construction of reduced models of composite slender structures was use. The elastic problem in a 3-D domain can be effectively separated in two independent sub-problems (cross-sectional and spanwise), corresponding to the different scales in the dimensions of the structure. The dynamics of the structure is recovered in the original 3-D domain, so that the interface of the structural module in the aeroelastic system is defined on the 3-D solid. In particular, this defines the deformed wetted surface in fluid-structure interaction problems. The reduced 1-D solution is simply regarded as an intermediate step in the solution process. Dealing with the slender structure from a 3-D perspective is a critical step in setting up high-fidelity aeroelastic simulations. Another important characteristic in this formulation (not exercised in this paper) is the 3-D recovery of the stress/strain fields through the solid. This gives an estimate of the maximum internal stresses in the structure for preliminary design. The compressible unsteady aerodynamic solver used to complete the aeroelastic formulation was ENS3DAE. Although both Euler and Navier-Stokes solutions are available, only the Euler one was exercised in the studies herein. Both the 3-D structural solver and the CFD solver were implemented as modules for MDICE, which managed the simulations and the interpolation of data between the two modules.

Numerical results were presented for the static aeroelastic analysis of a uniform wing (as a step towards dynamic aeroelastic simulations). There were different cases (isotropic and composites) representing different levels of elastic coupling in the structure. Since HALE vehicles tend to favor thick airfoils, transonic effects were shown to be present at uniform flow Mach number of less than 0.7. Moreover, results showed that, depending of the structural design, the inclusion of a more detailed representation of the wetted surface deformation becomes very important. Of particular importance may be deformation modes typically captured in 3-D built-up structures but not in classical beams. Results showed that the inclusion of a camber-bending degree of freedom can significantly shift the shock position and alter the flow around the wing. Therefore, the effect of higher-order deformations should be considered if 1-D structural models are used in a high-fidelity aeroelastic analysis, particularly in transonic flow. Finally, due to the large grid deformation involved in this nonlinear aeroelastic problem, a relaxation parameter was used to facilitate the convergence of the solution.

The proposed methodology was conceived for its use in high-fidelity nonlinear aeroelastic analysis of complex configurations, e.g., full aircraft transonic aeroelasticity, in the time domain. Further studies will focus on dynamic aeroelastic simulation.

\section{Acknowledgements}

The authors would like to sincerely thank Dr. Richard Snyder and Mr. Larry Huttsell (Air Force Research Laboratories) for the extensive technical interaction regarding ENS3DAE and MDICE. The ENS3DAE mesh of the slender wing provided by Prof. Marilyn Smith (Georgia Institute of Technology) is greatly appreciated. This work has been sponsored by NASA Langley Research Center (grant NAG-1-03038). The technical monitor is Dr. David M. Schuster.

\section{References}

1. Tilmann, C. P., Flick, P. M., Martin, C. A., and Love, M. H., "High-Altitude Long Endurance Technologies for SensorCraft," RTO AVT Symposium on Novel and Emerging Vehicle and Vehicle Technology Concepts. Brussels, Belgium, April 2003. RTO Paper MP-104-P-26, 2003.

2. Cosserat, E. and Cosserat, F., Théorie des corps déformables. A. Hermann et fils, Paris, 1909.

3. Love, A. E. H., Mathematical Theory of Elasticity. Dover, New York, 1944.

4. Reissner, E., “On One-Dimensional Large-Displacement Finite-Strain Beam Theory.” Studies in Applied Mathematics, Vol. 52, No. 2, pp. 87-95, 1973.

5. Simo, J. C., "Finite strain beam formulation: The three-dimensional dynamic problem - Part I." Computer Methods in Applied Mechanics and Engineering, Vol. 49, No. 1, pp. 55-70, 1985.

6. Hodges, D. H., “A Mixed Variational Formulation Based on Exact Intrinsic Equations for Dynamics of Moving Beams.” International journal of solids and structures, Vol. 26, No. 11, pp. 1253-1273, 1990. 
7. Garcia, J. A. and Guruswamy, G. P., “Aeroelastic Analysis of Transonic Wings Using Navier-Stokes Equations and a Nonlinear Beam Finite Element Model.” AIAA Paper 1999-1215, 1999.

8. Smith, M. J., Patil, M. J. and Hodges, D. H., “CFD-Based Analysis of Nonlinear Aeroelastic Behavior of High Aspect Ratio Wings.” AIAA Paper 2001-1582, 2001.

9. Cesnik, C. E. S. and Palacios, R., “Modeling Piezoelectric Actuators Embedded in Slender Structures.” AIAA Paper 2003-1803, 2003.

10. Palacios, R. and Cesnik, C. E. S., "Structural Dynamics of Integrally Strained Slender Wings." International Forum of Aeroelasticity and Structural Dynamics. Amsterdam, The Netherlands, June 2003.

11. Palacios, R. and Cesnik, C. E. S., "Reduced Structural Modeling of Integrally-Strained Slender Wings.” AIAA Paper 20042038, 2004.

12. Schuster, D. M., Vadyak, J. and Atta, E., "Static Aeroelastic Analysis of Fighter Aircraft Using a Three-Dimensional NavierStokes Algorithm.” Journal of Aircraft, Vol. 27, No. 9, pp. 820-825, 1990.

13. Kingsley, G., Siegel, J. M., Harrand, V. J., Lawrence, C. L. and Luker, J. J., "Development of a Multi-Disciplinary Computing Environment (MDICE).” AIAA Paper 1998-4738, 1998.

14. Smith, M. J., Hodges, D. H. and Cesnik, C. E. S., "Evaluation of Computational Algorithms Suitable for Fluid-Structure Interactions.” Journal of Aircraft, Vol. 37, No. 2, pp 282-294, 2000.

15. Cesnik, C. E. S. and Hodges, D. H., “VABS: ‘A New Concept for Composite Rotor Blade Cross-Sectional Modeling.” Journal of the American Helicopter Society, Vol. 42, pp 27-38, 1997.

16. Brown, S. A., “Displacement Extrapolation for CFD and CSM Aeroelastic Analysis.” AIAA Paper 97-1090, 1997. 